\title{
Gravitational Lensing and $f(R)$ theories in the Palatini approach
}

\author{
Matteo Luca RUGGIERO* \\ Dipartimento di Fisica, \\ Politecnico di Torino, Corso Duca degli Abruzzi 24, 10129 Torino \\ and INFN, Sezione di Torino
}

(Dated: October 27, 2018)

\begin{abstract}
We investigate gravitational lensing in the Palatini approach to the $f(R)$ extended theories of gravity. Starting from an exact solution of the $f(R)$ field equations, which corresponds to the Schwarzschild-de Sitter metric and, on the basis of recent studies on this metric, we focus on some lensing observables, in order to evaluate the effects of the non linearity of the gravity Lagrangian. We give estimates for some astrophysical events, and show that these effects are tiny for galactic lenses, but become interesting for extragalactic ones.
\end{abstract}

PACS numbers: 95.62.Sb, 04.50.Kd, 95.30.Sf

Keywords: Gravitational Lensing, Extended Theories of Gravity

\section{INTRODUCTION}

It is well known and accepted nowadays that the Universe is undergoing a phase of accelerated expansion [1]. General Relativity (GR), in its standard formulation, cannot explain this fact without requiring the existence of the so called dark energy, or introducing a cosmological constant which, in turn, brings about other problems, concerning its nature and origin 2]. According to a different viewpoint, current observations might suggest the failure of GR to describe gravitational interactions at cosmological scale: as a natural consequence, the claim arises for a modification of the theory of gravity. In recent years, there has been much interest for the so called $f(R)$ theories of gravity: in these theories, the gravitational Lagrangian depends on an arbitrary analytic function $f$ of the scalar curvature $R$ (see [3] and references therein). These theories are also referred to as extended theories of gravity, since they naturally generalize GR on a geometric ground: in fact, when $f(R)=R$ the action reduces to the usual Einstein-Hilbert action, and Einstein's theory is obtained. The field equations of these theories can be obtained in the metric formalism [4], by varying the action with respect to metric tensor, or in the Palatini formalism, where the action is varied with respect to the metric and the affine connection, which are supposed to be independent from each other [5]. The increasing interest for these theories is mainly due to the fact that it seems that they are able to provide cosmologically viable models (see [6] and references therein), in which both the late time acceleration and the inflation phase are present.

However, we must remember that even though there are problems in explaining the cosmological dynamics, GR is in excellent agreement with other gravity tests, such as Solar System ones and those coming from binary pulsar observations [7]; hence, as a matter of fact, every modified theory of gravity should reproduce GR in a suitable weak field limit. This issue is crucial for $f(R)$ theories: their weak field limits were largely analyzed, and the comparison with some known tests of gravity was carried out. A lively debated developed because some models have been ruled out [8], while other seem to pass Solar System tests [9]. Among the other things, stellar models have been investigated, and there is not a common agreement on the results. In fact, on the one hand, the Palatini approach seems to provide self consistent solutions [10], on the other hand, the same problem studied in the metric approach leads to unacceptable results [11] (however see [12]; see [13] for general difficulties with the Palatini approach). Gravitational lensing (i.e. the deflection of light rays due to the curvature of space-time), besides having an historical importance, since it gave one of the first confirmations of Einstein's theory in the famous eclipse of 1919, is very important today, because data coming from modern lensing observations are crucial to explore the Universe at very different scales [14, 15, 16]. It is then natural to ask whether modifications of the gravity Lagrangian, as those introduced in the $f(R)$ theories, affect the standard theory of lensing (where "standard" stands for "GR lensing") and, in particular, the interpretation of its phenomenology. Gravitational lensing in the framework of $f(R)$ was studied in [17]: in particular, theories with $f(R) \propto R^{n}$ were investigated in the metric approach, with emphasis on point-like lenses. In the present paper we want to address a similar task, but working in the Palatini approach (however, as we remark below, our results apply also to a subset of the solutions metric $f(R)$ gravity). In a previous work [18] we found an exact solution of the $f(R)$ field equations for the vacuum case, which corresponds to the Schwarzschild-de Sitter metric: starting from

*Electronic address: matteo.ruggiero@polito.it 
this solution and taking into account recent studies on this metric [19], we want to investigate the impact of the non linearity of the gravity Lagrangian on some lensing observables. The paper is organized as follows: in Section III we briefly review the Palatini approach to $f(R)$ theories, which lead to the Schwarzschild-de Sitter vacuum solution; in Section III we focus on point like-lensing, in GR (Schwarzschild space-time) and in $f(R)$ theories (Schwarzschild-de Sitter space-time). Finally, discussions and conclusions are outlined in Section IV

\section{A VACUUM EXACT SOLUTION OF THE $f(R)$ GRAVITY FIELD EQUATIONS}

The equations of motion of $f(R)$ gravity in the Palatini formalism can be obtained by independent variations with respect to the metric and the connection from the action ${ }^{1}$

$$
A=A_{\text {grav }}+A_{\text {mat }}=\int\left[\sqrt{g} f(R)+2 \kappa L_{\text {mat }}(\psi, \nabla \psi)\right] d^{4} x
$$

where $R \equiv R(g, \Gamma)=g^{\alpha \beta} R_{\alpha \beta}(\Gamma), R_{\mu \nu}(\Gamma)$ is the Ricci-like tensor of any torsionless connection $\Gamma$ independent from the metric $g$, which is assumed here to be the physical metric. The gravitational part of the Lagrangian is represented by any real analytic function $f(R)$ of the scalar curvature $R$. The matter Lagrangian $L_{\text {mat }}$ is functionally depending on unspecified matter fields $\Psi$ together with their first derivatives, equipped with a gravitational coupling constant $\kappa=\frac{8 \pi G}{c^{4}}$.

According to the Palatini formalism [5], from (10) we obtain the following equations of motion:

$$
\begin{aligned}
f^{\prime}(R) R_{(\mu \nu)}(\Gamma)-\frac{1}{2} f(R) g_{\mu \nu} & =\kappa T_{\mu \nu}^{m a t}, \\
\nabla_{\alpha}^{\Gamma}\left[\sqrt{g} f^{\prime}(R) g^{\mu \nu}\right) & =0,
\end{aligned}
$$

where $T_{\text {mat }}^{\mu \nu}=-\frac{2}{\sqrt{g}} \frac{\delta L_{\text {mat }}}{\delta g_{\mu \nu}}$ denotes the matter source stress-energy tensor and $\nabla^{\Gamma}$ means covariant derivative with respect to the connection $\Gamma$. The equations of motion (2) can be supplemented by the scalar-valued equation obtained by taking the $g$-trace of (2), where we set $\tau=\operatorname{tr} T=g^{\mu \nu} T_{\mu \nu}^{m a t}$ :

$$
f^{\prime}(R) R-2 f(R)=\kappa \tau \text {. }
$$

The algebraic equation (4) is called the structural equation and it controls the solutions of equations (2).

The field equations (2,3) and the structural equation (4) in vacuum become

$$
\begin{aligned}
{\left[f^{\prime}(R)\right] R_{(\mu \nu)}(\Gamma)-\frac{1}{2}[f(R)] g_{\mu \nu} } & =0 \\
\nabla_{\alpha}^{\Gamma}\left(\sqrt{g}\left[f^{\prime}(R)\right] g^{\mu \nu}\right) & =0 \\
f^{\prime}(R) R-2 f(R) & =0 .
\end{aligned}
$$

As shown in [18] (see, in particular, Section 3), the system of equations (50 7) has the spherical symmetrical solution

$$
d s^{2}=-b(r) d t^{2}+\frac{d r^{2}}{b(r)}+r^{2} d \vartheta^{2}+r^{2} \sin ^{2} \vartheta d \varphi^{2}
$$

where $b(r) \doteq\left(1-\frac{2 M}{r}-\frac{k r^{2}}{3}\right) ; M$ is the mass of the source of the gravitational field and $k=-c_{i} / 4$, where $R=c_{i}$ is any of the solutions of the structural equation (7). In doing so, we have obtained a solution with constant scalar curvature $R$. In particular, if $f(R)=R$ (i.e. our theory is GR) then $R=0$ is the solution of the structural equation, and (8) reduces to the classical Schwarzschild solution.

Remark. We would like to point out that, for a given $f(R)$ function, in vacuum case the solutions of the field equations of Palatini $f(R)$ gravity are a subset of the solutions of the field equations of metric $f(R)$ gravity [20]. In

\footnotetext{
${ }^{1}$ Let the signature of the 4-dimensional Lorentzian manifold $M$ be $(-,+,+,+)$; furthermore, if not otherwise stated, we use units such that $G=c=1$.
} 
particular the spherically symmetric solution (8) is also a solution of the field equations of metric $f(R)$ gravity with constant scalar curvature $R$ : actually, in metric $f(R)$ gravity there are also spherically symmetric solutions where the scalar curvature is not constant (see e.g. [21] ). So, strictly speaking, what follows applies also to metric $f(R)$ gravity.

In GR, the Schwarzschild-de Sitter (8) solution corresponds to a spherically symmetric solution of Einstein field equations, with a cosmological term $\Lambda g_{\mu \nu}, \Lambda$ being the cosmological constant. In practice, it is $\Lambda=k$ in our notation. As we said, the cosmological constant is one of the candidates for explaining the accelerated expansion of the Universe (in particular, a positive cosmological constant is required). The data suggest that it cannot exceed the upper value of $\Lambda_{0} \simeq 10^{-52} m^{-2}$ [22]. As a consequence, it is reasonable to estimate that the solutions of the structural equation have the same order of magnitude; the same holds for the parameter $k$ in the metric (8).

In a recent paper [19] it has been shown that, contrary to previous claims (e.g. [23]), the $k$ terms in (8) does contribute to the observed bending of light rays (see also the subsequent papers [24], [25], [26], [27]). The key point is that the Schwarzschild-de Sitter space-time does not become flat at spatial infinity and, then, the spatial geometry affects deflection measurements.

In our approach, the parameter $k$ is ultimately related to the non linearity of the action, in fact when $f(R)=R \rightarrow$ $k=0$. Hence, the study of the effects of the $k$ term on light bending can be interpreted as an investigation of the effects of the non linearity of the gravity Lagrangian, which is what we want to address here.

According to [19], the bending angle of a light ray in the gravitational field of a massive object described by (8), turns out to be

$$
\varepsilon_{S d S}=\frac{4 M}{\rho}\left(1-\frac{k \rho^{4}}{24 M^{2}}\right),
$$

up to first order in both $M$ and $k$, if both the source of light rays and the observer are far from the massive object (the lens). We see that, when $k=0$, i.e. in GR, $\epsilon_{S d S}$ reduces to the classical bending angle $\varepsilon_{G R}=\frac{4 M}{\rho}$ (e.g. [28]). The parameter $\rho$, to lowest order in $M$ and $k$ is equivalent to the impact parameter. In what follows, we will investigate gravitational lensing on the basis of the bending angle (9).

\section{LENSING FROM POINT-LIKE SOURCES}

In this Section, we start by briefly reviewing the basic notions of gravitational lensing from a spherically symmetrical point-like source in GR, i.e. we consider lensing in the Schwarzschild field (for a thorough study of Schwarzschild lensing, see e.g. [29]) Then, we consider the case of $f(R)$ theories which, on the basis of what we have said before, corresponds to studying lensing in the Schwarzschild-de Sitter space-time.

\section{A. Basics of lensing from a spherically symmetrical source}

In Figure 1 a simplified lensing geometry is depicted. The observer in $O$ sees the image of the source, located at $S$, as if it were in $S_{1}$. The lens is located at the point $L$. In the so called thin lens approximation (see [14, 15, 16]) the light paths are approximated by straight lines; if the lens has spherical symmetry, which is what we assume here, light rays propagate in a plane. We may write the following relation (see e.g. [29]), which links the angular positions of the images $(\vartheta)$, the actual position of the source $(\beta)$ and the bending angle $(\varepsilon)$

$$
D_{s} \tan \vartheta=D_{s} \tan \beta+D_{d s} \tan \varepsilon
$$

and, since $D_{s} \tan \alpha=D_{d s} \tan \varepsilon$ :

$$
D_{s} \tan \vartheta=D_{s} \tan \beta+D_{s} \tan \alpha
$$

So, if we assume that the angles are small (weak lens approximation), we are able to write the following lens equation

$$
\beta=\vartheta-\alpha(\vartheta)
$$




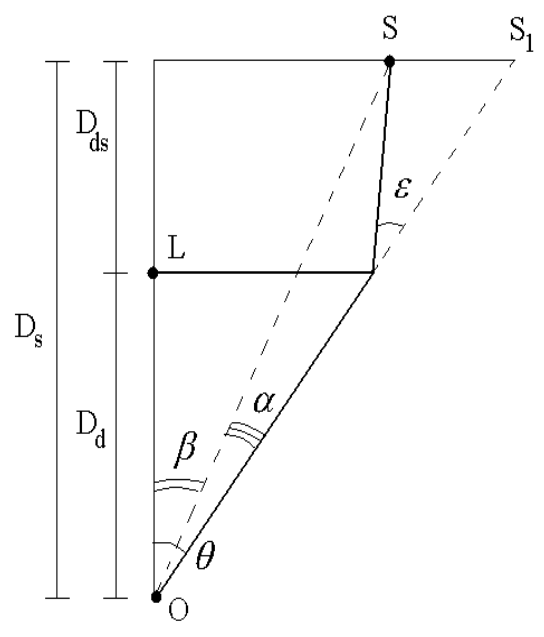

FIG. 1: A simplified lensing geometry: light paths are depicted as straight lines. The observer in $O$ sees the image of the source, located at $S$, as if it were in $S_{1}$. The lens is located at the point $L$. Distances between the observer and the source, the observer and the lens and the lens and the source are, respectively, defined by $D_{s}, D_{d}, D_{d s}$.

The latter equation allows to obtain the angular positions of the images once those of the sources and the bending angle are known. On using back the fact that $\alpha D_{s} \simeq \varepsilon D_{d s}$, we may write

$$
\beta=\vartheta-\frac{D_{d s}}{D_{s}} \varepsilon(\vartheta)
$$

in which the deflection angle $\varepsilon$ appears.

For instance, in the case of lensing in the Schwarzschild gravitational field

$$
d s^{2}=-a(r) d t^{2}+\frac{d r^{2}}{a(r)}+r^{2} d \vartheta^{2}+r^{2} \sin ^{2} \vartheta d \varphi^{2},
$$

where $a(r) \doteq\left(1-\frac{2 M}{r}\right)$. The point-like lens is a spherical object of mass $M$; the bending angle turns out to be $\varepsilon=\varepsilon_{G R}=4 M / \rho$, where $\rho=D_{d} \vartheta$ is the impact parameter. On substituting, we get the explicit form of the lens equation in this case

$$
\beta=\vartheta-\frac{D_{d s}}{D_{s} D_{d}} \frac{4 M}{\vartheta} .
$$

The position corresponding to $\beta=0$, which describes the alignment of observer, lens and source, defines the so called Einstein angle

$$
\vartheta_{E} \doteq \sqrt{4 M \frac{D_{d s}}{D_{s} D_{d}}}
$$

which allows to define the characteristic length scale of lensing

$$
R_{E}=\vartheta_{E} D_{d},
$$

which is called Einstein radius. On introducing the Einstein angle in the lens equation (15), we get

$$
\beta \vartheta=\vartheta^{2}-\vartheta_{E}^{2},
$$

or expressing angles in units of the Einstein angle $\vartheta_{E}$, i.e. setting $\beta=\bar{\beta} \vartheta_{E}, \vartheta=\bar{\vartheta} \vartheta_{E}$, the lens equation has the following expression

$$
\bar{\beta} \bar{\vartheta}=\bar{\vartheta}^{2}-1
$$


The latter equation can be simply solved to give the angular positions of the images

$$
\bar{\vartheta}_{ \pm}=\frac{\bar{\beta} \pm \sqrt{\bar{\beta}^{2}+4}}{2}
$$

and the angular separation between the two images

$$
\Delta \bar{\vartheta} \doteq \bar{\vartheta}_{+}-\bar{\vartheta}_{-}=\sqrt{\bar{\beta}^{2}+4} .
$$

\section{B. Lensing in the Schwarzschild-de Sitter field}

In order to obtain the explicit expression of the lens equation in the Schwarzschild-de Sitter space-time, we have to introduce the bending angle (9) in eq. (13). So, taking into account that $\rho=D_{d} \vartheta$, we may write

$$
\beta=\vartheta-\frac{D_{d s}}{D_{s} D_{d}} \frac{4 M}{\vartheta}+\frac{D_{d s} D_{d}^{3}}{D_{s}} \frac{\vartheta^{3} k}{6 M} .
$$

On introducing the following dimensionless parameter

$$
\chi \doteq \frac{2}{3} k \frac{D_{d s}^{2} D_{d}^{2}}{D_{s}^{2}},
$$

and expressing angles in units of the Einstein angle $\vartheta_{E}$ defined by (16), the lens equation becomes

$$
\bar{\beta} \bar{\vartheta}=\bar{\vartheta}^{2}-1+\bar{\vartheta}^{4} \chi .
$$

The lens equation (24) differs from the GR one (19) because of the term proportional to $\chi$ which, in turn, is proportional to the parameter $k$, related to the non linearity of the gravity Lagrangian. Indeed, we notice that, beyond the parameter $k, \chi$ depends on the geometry of lensing, through the distances $D_{d s}, D_{d}, D_{s}$, which enter its definition; $\chi$ is reasonably small in actual astrophysical events, chiefly because of the smallness of the parameter $k$, whose expected order of magnitude is the one of the cosmological constant (see Section II). As a consequence, the term $\bar{\vartheta}^{4} \chi$ can be treated as a perturbation with respect to the GR lens equation (19).

In what follows, we are going to analyze some observables in gravitational lensing, in order to evaluate whether the non linearity of the Lagrangian (1) raises detectable effects.

To begin with, we calculate the solutions of the lens equation (24) for the case $\bar{\beta}=0$, which corresponds to perfect alignment of observer, lens and source (superior conjunction): in other words, we look for the modified Einstein angle in $f(R)$ theories.

In what follows we use $\vartheta_{E, G R}$ to refer to the standard Einstein angle (16), i.e. the one obtained in GR, for a spherically symmetrical point-like lens (described by the gravitational field (14)), while we use $\vartheta_{E, E T G}$ to indicate the Einstein angle in $f(R)$ for a spherically symmetrical point-like lens (described by the field (88)). Furthermore, we write angles in units of $\vartheta_{E, G R} \doteq \vartheta_{E}$, defined in (16).

We look for a solution of the equation

$$
0=\bar{\vartheta}^{2}-1+\bar{\vartheta}^{4} \chi
$$

in the form

$$
\bar{\vartheta}_{E, E T G}=1+\delta,
$$

where $\delta$ is assumed to be small, i.e. of first order in $\chi$. We then obtain

$$
\delta=-\frac{\chi}{2}
$$

Hence we have, up to first order in $\chi$

$$
\frac{\vartheta_{E, E T G}-\vartheta_{E, G R}}{\vartheta_{E, G R}}=-\frac{\chi}{2} .
$$

In particular, we see from (27) that the modified Einstein angle $\vartheta_{E, E T G}$ is smaller (bigger) than the classical one $\vartheta_{E, G R}$ if $\chi$ is positive (negative): this means that, according to (23), a positive (negative) value of $k$ decreases 
(increases) the Einstein angle. If we recall that $k$ can be interpreted as a cosmological constant (see Section II), and we remember that it is required to be positive to mimic the accelerated expansion of the universe, we conclude that in this approach to $f(R)$ we obtain an Einstein angle that is smaller than the standard GR one: this is indeed independent on the analytic form of the $f(R)$, and depends on the solutions of the structural equation only.

We continue our analysis by taking into account the lensing-induced magnification: actually, gravitational lenses can amplify the luminosity of the sources, so that faint sources can become visible. In the case of point-like lenses, the magnification turns out to be [15]

$$
A \doteq\left|\frac{\beta}{\vartheta} \frac{d \beta}{d \vartheta}\right|^{-1}
$$

Then, from the lens equation (24) we obtain, up to first order in $\chi$

$$
A=\left|1-\frac{1}{\vartheta^{4}}+2 \chi\left(2 \vartheta^{2}-1\right)\right|^{-1}
$$

We see that when $\chi=0$ the GR result $A=\left|1-\frac{1}{\vartheta^{4}}\right|^{-1}$ is recovered. Actually, in observations what is seen is the total luminosity, i.e. the sum of the magnification of each image. However, in practice, it is not always possible to see the (multiple) images of the sources, together with their magnification, but the magnification can be detected thanks to the relative motion of the lens and the source, that gives rise to a lensing-induced time variability in the magnification. The time scaling for these variations can be estimated by $t_{E}=R_{E} / v=D_{d} \vartheta_{E} / v$, where $v$ is a typical velocity of the lens (transverse to the line of sight). Consequently, a variation of the Einstein angle provokes a variation of this time scale. If we set $t_{E T G}=D_{d} \vartheta_{E T G} / v$, according to what we have seen, then, we can estimate the variation as

$$
\frac{\Delta t}{t_{E}}=\frac{t_{E T G}-t_{E}}{t_{E}}=\delta=-\frac{\chi}{2} .
$$

Let us now focus on the estimate of the lens mass, determined by lensing phenomenology: if we suppose that the distances and the transverse velocity are known, a measurement of $t_{E}$ gives an estimate of the mass $M$ of the lens. As a consequence if we "incorrectly" use the Einstein angle of GR instead of the $f(R)$ one, we get a different (biased) mass of the lens. If we denote as $M$ the "true" mas of the lens, and as $M_{G R}$ the one obtained by using the GR expression of the Einstein angle, we get

$$
\frac{M_{G R}-M}{M}=2 \delta=-\chi
$$

So, according to what we have said above, the effects of $f(R)$ is an underestimate of the lens mass, in other words the true lens mass is bigger than the one estimated in standard GR lensing.

Another phenomenological issue that can be investigated is how the positions of the images (20) are modified in $f(R)$ theories of gravitation. Since $\chi$ is reasonably small, the lens equation (24) can be solved perturbatively: in other words we look for the lowest order corrections of the $k$ term on the GR solutions. To this end, let $\bar{\vartheta}_{0}$ be a solution of the lens equation corresponding to $\chi=0$ :

$$
\bar{\beta} \bar{\vartheta}_{0}=\bar{\vartheta}_{0}^{2}-1 .
$$

In particular, $\bar{\vartheta}_{0}$ is equal to the solutions (20) given above. Then, we look for solutions in the form

$$
\bar{\vartheta}=\bar{\vartheta}_{0}+\bar{\vartheta}_{1}
$$

where $\bar{\vartheta}_{1}$ is supposed to be small. On substituting (34) in (24), and linearizing in $\bar{\vartheta}_{1}$, we obtain, up to first order in $\chi$

$$
\bar{\vartheta}_{1}=-\frac{\bar{\vartheta}_{0}^{4}}{2 \bar{\vartheta}_{0}-\bar{\beta}} \chi
$$

As a consequence, we may write

$$
\bar{\vartheta}_{ \pm}=\bar{\vartheta}_{0 \pm}-\frac{\bar{\vartheta}_{0 \pm}^{4}}{2 \bar{\vartheta}_{0 \pm}-\bar{\beta}} \chi
$$




\begin{tabular}{ccc|c}
$D_{d}[K p c]$ & $D_{s}[K p c]$ & $D_{d s}[K p c]$ & $\chi$ \\
\hline 20 & 50 & 30 & $8.6 \times 10^{-10}$ \\
7.6 & 7.6 & $10^{-3}$ & $6.0 \times 10^{-18}$ \\
7.6 & 50 & 40 & $2.5 \times 10^{-10}$ \\
$5 \times 10^{3}$ & $10^{4}$ & $10^{4}$ & $1.5 \times 10^{-4}$ \\
\hline
\end{tabular}

TABLE I: Evaluation of the dimensionless parameter $\chi$ (which parameterizes the deviation of the $f(R)$ theories from GR) as a function of the distances between the observer and the lens, the observer and the source and the lens and the source, respectively defined by $D_{d}, D_{s}, D_{d s}$ (all measured in $K p c$ ); the value of the parameter $k$ is $10^{-52} \mathrm{~m}^{-2}$. form

If we set $\Delta \bar{\vartheta}_{0}=\sqrt{\bar{\beta}^{2}+4}$ according to (21), we may write the angular separation between the two images in the

$$
\Delta \bar{\vartheta}=\Delta \bar{\vartheta}_{0}-g\left(\Delta \bar{\vartheta}_{0}\right) \chi
$$

where

$$
g\left(\Delta \bar{\vartheta}_{0}\right) \doteq\left[\left(\sqrt{\Delta \bar{\vartheta}_{0}^{2}-4}+\Delta \bar{\vartheta}_{0}\right)^{4}+\left(\sqrt{\Delta \bar{\vartheta}_{0}^{2}-4}-\Delta \bar{\vartheta}_{0}\right)^{4}\right] \frac{1}{16 \Delta \bar{\vartheta}_{0}}
$$

In our approach, the modifications (28), (30), (31), (32), (37) determined by the non linearity of the gravity Lagrangian consist in terms that are proportional to the parameter $\chi$, which is what one would expect in a first order approximation. As a consequence, the evaluation of the detectability of these modifications requires an estimate of the parameter $\chi$ in some actual astrophysical events, which will be done in the following Section.

\section{DISCUSSION AND CONCLUSIONS}

We have evaluated some effects of the non linearity of the gravity Lagrangian on lensing phenomenology, in the Palatini approach to $f(R)$ extended theories of gravity, however our results apply also to a subset of solutions of metric $f(R)$ gravity.

These effects are controlled by the parameter $\chi$; the latter is proportional to $k$ which, in turn, is simply related to the solution of the structural equation (7) and it parameterizes the deviation of the $f(R)$ theories from GR: namely, when $k=0$, GR is recovered. The parameter $\chi$ depends also on the lens geometry, through the distances $D_{d s}, D_{d}, D_{s}$, which enter its definition (23); $\chi$ is expected to be small, because of the smallness of the parameter $k$, whose order of magnitude is comparable to the cosmological constant. Taking into account this fact, we have evaluated the $f(R)$ effects as perturbations of the standard GR results. To quantitatively evaluate these effects it is necessary to give numerical estimates of the dimensionless parameter $\chi$ in some astrophysical events. Taking into account some reasonable values for lenses in our galaxy, we obtain numerically:

$$
\chi \simeq 1.5 \times 10^{-10}\left(\frac{k}{10^{-52} m^{-2}}\right)\left(\frac{D_{d s}}{10 K p c}\right)^{2}\left(\frac{D_{d}}{10 K p c}\right)^{2}\left(\frac{20 K p c}{D_{s}}\right)^{2} .
$$

To fix the ideas, we can analyze some specific events. To begin with, let us consider a lens in the galactic halo, with $D_{d}=20 \mathrm{Kpc}$, and and extra galactic source at $D_{s}=50 \mathrm{Kpc}$ : we obtain $\chi=8.6 \times 10^{-10}$. Then, we can consider the supermassive black hole in the radio source Sgr $\mathrm{A}^{*}$, with $D_{d}=7.6 \mathrm{Kpc}$, and a source (1) just behind the black hole $D_{s}=1 p c$, (2) outside the galaxy $D_{s}=50 \mathrm{Kpc}$ we get, respectively: $(1) \chi=6.0 \times 10^{-18},(2) \chi=2.5 \times 10^{-10}$.

In general, for sources outside the galaxy we may set $D_{s} \sim D_{d s}$; consequently if we think of distant galaxies (or cluster of galaxies) acting as lenses, we obtain numerically

$$
\chi \simeq 6.0 \times 10^{-4}\left(\frac{k}{10^{-52} m^{-2}}\right)\left(\frac{D_{d}}{10 M p c}\right)^{2} .
$$


For instance if we consider $D_{d}=5 M p c$, we obtain $\chi=1.5 \times 10^{-4}$. These estimates are summarized in Table 【.

We remember that the order of magnitude of the lensing phenomena is roughly determined by the Einstein angle; according to eq. (28) modifications of the Einstein angle due to the non linearity of the gravity Lagrangian are proportional to $\chi \vartheta_{E, G R}$. As a consequence, the smallness of $\chi$ makes the effects of $f(R)$ theories on lensing hardly detectable for galactic lenses. The situation is different for extragalactic lenses: in fact, in this case we have $\chi \vartheta_{E, G R} \propto$ $M^{1 / 2} D_{d}^{3 / 2}$, so that distant galaxies or clusters of galaxies acting as lenses can be interesting for our purposes. We notice that similar results have been obtained in [24] where, working in the GR framework, the effects of the cosmological constant on lensing by distant clusters of galaxies have been evaluated. However, in the case of extragalactic sources, i.e. when the lenses are galaxies or clusters of galaxies, the point-like lens model that we have used in this paper might be oversimplified: a more realistic model is needed in order to investigate the possibility of detecting these effects by means of the present and foreseeable observational techniques.

Nonetheless the estimates that we have obtained suggest at least the scale where the impact of the $f(R)$-induced corrections on lensing phenomenology becomes interesting.

To summarize, the non linear terms in the gravity action determine a modification of lensing phenomenology; numerically, for actual astrophysical events, these modifications are very small if we deal with lenses in our galaxy: these results suggest that the effect of $f(R)$ are confined around a cosmological scale and, hence, they are not effective at the galactic scale. On the contrary, in our simple model, extragalactic lenses lend themselves as natural candidates for studying the effects of extended theories of gravity. However, more realistic models and further studies are needed to evaluate the detectability of these effects.

Acknowledgments. The author acknowledges financial support from the Italian Ministry of University and Research (MIUR) under the national program "Cofin 2005" - La pulsar doppia e oltre: verso una nuova era della ricerca sulle pulsar.

[1] Riess A. G. et al., Astron. J. 116, 1009 (1998); Perlmutter S. et al., Astroph. J. 517, 565 (1999); Tonry J.L. et al., Astroph. J. 594, 1(2003); Bennet, C.L. et al, Astroph. J. Suppl. 148, 1 (2003); Netterfield et al., Astroph. J. 571, 604 (2002); Halverson N.W. et al., Astroph. J. 568, 38 (2002)

[2] Peebles P.J.E., Ratra B., Rev. Mod. Phys.75, 559 (2003)

[3] Capozziello S., Francaviglia M., arXiv:0706.1146 (2007); Sotiriou T.P., arXiv:0710.4438. (2007); Sotiriou, T., Faraoni, V., arXiv:0805.1726 (2008)

[4] H.A. Buchdahl, Month. Not. R. Astron. Soc. 150, 1 (1970); Nojiri S., Odintsov S.D., Phys. Rev. D 68, 123512 (2003); Nojiri S., Odintsov S.D., Gen. Rel. Grav. 36, 1765 (2004); Nojiri S., Odintsov S.D., Mod. Phys. Lett. A 19, 627 (2004); Olmo G. J., Phys. Rev. D 72, 083505 (2005)

[5] Ferraris M., Francaviglia M., Volovich I., Nuovo Cim. B 108, 1313 (1993); Ferraris M., Francaviglia M., Volovich I., Class. Quant. Grav. 11, 1505 (1994); Barraco D., Hamity V. H., Vucetich H., Gen. Rel. Grav. 34, 533 (2002); Vollick D.N., Phys. Rev. D 68, 063510 (2003); Olmo G.J., Phys. Rev. Lett. 95 (2005) 261102; Olmo G.J., Komp W., arXiv:gr-qc/0403092 (2004); Meng X.H., Wang P., Class. Quant. Grav. 20, 4949 (2003); Meng X.H., Wang P., Class. Quant. Grav. 21, 951 (2004); Kremer G.M., Alves D.S.M., Phys.Rev. D 70, 023503 (2004)

[6] Capozziello S., Carloni S. Troisi A., [arXiv:astro-ph/030304]; Allemandi G., Borowiec A., Francaviglia M, Phys. Rev. D 70, 043524 (2004); Sotiriou T.P., Class. Quant. Grav. 23, 1253 (2006); Tsujikawa S., arXiv:0709.1391] (2007), Nojiri S., Odintsov S.D., arXiv:0707.1941] (2007); Starobinski A., JETP Lett. 86, 157 (2007); Amendola L., Tsujikawa S., arXiv:0705.0396. (2007)

[7] Will C.M., Living Rev. Relativity 9, 3 (2006), http://www.livingreviews.org/lrr-2006-3

[8] Chiba T., Phys. Lett. B 575, 1 (2003); Olmo G. J. , Phys. Rev. D 75, 023511 (2007); Chiba T., Smith T.L., Erickcek A.L., Phys. Rev. D 75, 124014 (2007)

[9] Nojiri S., Odintsov S.D., arXiv:0710.1738 (2007); Hu W., Sawicki I, arXiv:0705.1158 (2007); Faraoni V., Phys. Rev. D 74, 023529 (2006); Sotiriou T.P., Gen. Rel. Grav. 38, 1407 (2006); Faulkner T., Tegmark M., Bunn E. F., Mao Y., arXiv:astro-ph/0612569. (2006); Ruggiero M.L., Iorio L., JCAP 0701010 (2007); Allemandi G., Ruggiero M.L., Gen. Rel. Grav. 39, 1381 (2007)

[10] Kainulainen K., Reijonen V., Sunhede D., arXiv:gr-qc/0611132 (2006), Kainulainen K., Piilonen J., Reijonen V., Sunhede D., arXiv:0704.2729] (2007)

[11] Erickcek A.L., Smith T. L. , Kamionkowski M., Phys. Rev. D 74, 121501 (2006); Multamaki T., Vilja I., arXiv:0709.3422 (2007)

[12] Barausse E., Sotiriou T.P., Miller J.C., arXiv:gr-qc/0703132 (2007); Barausse E., Sotiriou T.P., Miller J.C., arXiv:0712.1141 (2007)

[13] Iglesias A., Kaloper N., Padilla A., Park M., arXiv:0708.1163] (2007)

[14] Petters A.O., Levine H., Wambsganss J., Singularity Theory and Gravitational Lensing, Birkäuser, Basel (1998) 
[15] Schneider P., Ehelers J., Falco E.E., Gravitational Lenses, Springer, Berlin (1992)

[16] Wambsganss J., Living Rev. Relativity 1, 12 (1998) http://www.livingreviews.org/lrr-1998-12

[17] Capozziello S., Cardone V.F., Troisi A., Phys. Rev. D 73, 104019 (2006)

[18] Allemandi G., Francaviglia M., Ruggiero M.L., Tartaglia A., Gen. Rel. Grav. 37, 1891 (2005)

[19] Rindler W., Ishack M., Phys. Rev. D76 (2007) 043006,

[20] Magnano G., Talk given at the XI Italian Conference on General Relativity and Gravitation Trieste, Sept 2630, 1994, arXiv:gr-qc/9511027. (1995); Allemandi G., Borowiec A., Francaviglia M., Odintsov S.D., Phys.Rev. D 72, 063505 (2005)

[21] Multamaki T., Vilja I., arXiv:astro-ph/0606373 (2006)

[22] Kochanek C.S. , Astroph. J. 466, 638 (1996)

[23] Lake K., Phys. Rev. D 65, 087301 (2002)

[24] Ishak M., Rindler W., Dossett J., Moldenhauer J., Allison C., arXiv:0710.4726 (2007)

[25] Sereno M., arXiv:0711.1802] (2007)

[26] Lake K., arXiv:0711.0673] (2007)

[27] Schücker T., arXiv:0712.1559] (2007)

[28] Straumann N., General Relativity, Springer, Berlin (2004)

[29] Virbhadra K. S. , Ellis G.F.R., Phys. Rev. D 62, 084003 (2000) 